This document is the Accepted Manuscript version of a Published Work that appeared in final form in J. Chem. Phys. 130, 124319 (2009), copyright $(c)$ American Institute of Physics after peer review and technical editing by the publisher. To access the final edited and published work see

jcp.aip.org 


\title{
Interior- and Surface-Bound Excess Electron States in Large Water Cluster Anions
}

\author{
Ádám Madarász ${ }^{\mathrm{a}, \mathrm{c}}$, Peter J. Rossky ${ }^{\mathrm{b}, \mathrm{c}}$ and László Turi ${ }^{\mathrm{a}, \mathrm{c}}$ \\ ${ }^{a}$ Eötvös Loránd University, Department of Physical Chemistry, Budapest 112, P. O. Box 32, \\ H-1518, Hungary \\ ${ }^{\mathrm{b}}$ Department of Chemistry and Biochemistry and Institute for Computational Engineering and \\ Sciences, \\ University of Texas at Austin, Austin, TX 78712
}

We present the results of mixed quantum/classical simulations on relaxed thermal nanoscale water cluster anions, $\left(\mathrm{H}_{2} \mathrm{O}\right)_{n}^{-}$, with $n=200,500,1000$ and 8000. By using initial equilibration with constraints, we investigate stable/metastable negatively charged water clusters with both surface-bound and interior-bound excess electron states. Characterization of these states is performed in terms of geometrical parameters, energetics, and optical absorption spectroscopy of the clusters. The calculations provide data characterizing these states in the gap between previously published calculations, and experiments, on smaller clusters and the limiting cases of either an excess electron in bulk water, or an excess electron at an infinite water/air interface. The present results are in general agreement with previous simulations and provide a consistent picture of the evolution of the physical properties of water cluster anions with

\footnotetext{
${ }^{\mathrm{c}}$ E-mail: turi@chem.elte.hu, fax: (36)-1-372-2592

madaradam@gmail.com, fax: (36)-1-372-2592

rossky@mail.utexas.edu, fax: (1)-512-471-1624
} 
size over the entire size range, including results for vertical detachment energies and absorption spectra that would signify their presence. In particular, the difference in size dependence between surface-bound and interior-bound state absorption spectra is dramatic, while for detachment energies the dependence is qualitatively the same. 


\section{Introduction}

The physics of water cluster anions has remained an important focus of research efforts for more than two decades. The intense scientific interest is largely motivated by the central role of water cluster anions in various important physical processes in atmospheric chemistry, interstellar chemistry, and in electron-initiated processes in aqueous systems. ${ }^{1}$ Furthermore, the finite size and the anticipated relative simplicity of water cluster anions, compared to a condensed phase ${ }^{1}$ renders them an excellent model for both experimental ${ }^{2,3,4,5,6,7,8,9,10}$ and theoretical methods under welldefined conditions. ${ }^{11,12,13,14}$

Despite the considerable effort invested, there is still no consensus on the most basic structural properties of water cluster anions. Two distinct localization patterns have been predicted by theory for water cluster anions. In the interior-bound (IB) states, the excess electron localizes in a solvent void surrounded by properly oriented water molecules in clear analogy to the hydrated electron. ${ }^{11-14,15,16,17,18,19,20}$ Theoretical work also suggested that an alternative binding motif may exist with the excess electron being stabilized by the electrostatic reaction field of the cluster dipole, in surface-bound (SB) states, with significant electronic amplitude appearing outside the molecular framework. ${ }^{11-20}$

Sophisticated experiments on size selected clusters, however, observe at least three characteristic cluster anion classes reflected by three distinctly different trends in the variation of the vertical electron detachment energy with size. ${ }^{8,9,21}$ The systematic trends clearly suggest a commonality in the structural pattern within each class. A main challenge in the field is to expose the source of this regularity in the properties of the "isomeric" species behind the observed patterns. ${ }^{2-9,22,23,24,25,26,27,28}$ Based mainly on the limiting behavior of the physical properties extrapolated to 
infinite size, the strongest binding clusters have been thought to be IB clusters, ${ }^{9,21,22,23,}$ ${ }^{25}$ while the other two classes were postulated as different types of SB clusters. ${ }^{9,25,28}$ This interpretation has been challenged by the results of quantum molecular dynamics simulations which suggest that the properties of surface electronic states can be consistent with the experimental tendencies for the strongest binding cluster type. ${ }^{13,29}$ Specifically, it was shown that the computed trends in the radius and the kinetic energy of the excess electron with cluster size for SB clusters reproduce the tendencies extracted from experiments. ${ }^{30}$ Up to now, this is the strongest (and most intriguing) evidence in favor of strong surface-bound excess electronic states in water cluster anions. ${ }^{13,29}$ Several recent ab initio quantum chemical studies have also appeared in the literature adding substantially to the debate on both sides. ${ }^{14,15,17,18}$ One, however, must realize that static quantum chemistry results must be interpreted with caution. Both the computational methods and the choice of the investigated structures are of crucial importance, as was clearly pointed out by Sommerfeld. ${ }^{19}$ Also important is the fact that ensemble entropy is often not accounted for in such studies.

The major difficulty in interpreting the experimental signals lies in the fact that the different types of clusters are prepared under different experimental conditions, ${ }^{6,9,31}$ in particular, source backing pressure. ${ }^{6,9}$ It is generally reasonably assumed that higher backing pressure corresponds to colder experimental cluster conditions. ${ }^{9,32}$ However, it is also widely appreciated that the conditions are expected to be non-thermal, ${ }^{3,10}$ so that it is clearly difficult to characterize a cluster ensemble simply or unambiguously. On the other hand, theory, especially quantum molecular dynamics simulations generally assume thermal equilibrium of the cluster anion species. In this uncertain situation, there are alternative rational theoretical approaches. The route which is pursued almost exclusively is to locate and 
characterize stable isomers of water cluster anions by performing either static (i.e. quantum chemistry) or dynamic (i.e. molecular dynamics) calculations. Although these calculations usually sample only the dominant, most stable minima of the thermal equilibrium conditions, they provide important insight into the energetic and structural aspects of cluster anions. We consider the behavior of large anionic water clusters via this approach in the present paper. Keeping in mind that the clusters which are formed and detected in experiments may be far from equilibrium, it would be an equally important challenge to "simulate" and characterize metastable cluster anions that potentially are not substantially populated at equilibrium. We will pursue this problem in a subsequent paper.

In the present study, we specifically examine the properties of relaxed quasiequilibrium nanoscale ( $n \sim 200-8000)$ cluster anions. In particular, we investigate whether there exist (meta)stable states of negatively charged water clusters with surface-bound and/or interior-bound excess electrons. For those states that persist for at least $\sim 50 \mathrm{ps}$, we can evaluate their signature in various observables. These cluster anions are larger than those so far investigated in detail experimentally for water, or considered in previous computational work. This study, thus, attempts to extend a guide as to the cluster size dependence which may be observable in experiments and be identified with SB or IB electronic states. In the process, we collect and compile all of our previous and present simulation results, which then completely span from fairly small $(n=20)$ cluster sizes to infinite size surface-bound interfacial and interiorbound bulk hydrated electrons.

\section{Methods}


We performed mixed quantum-classical molecular dynamics simulation of nanoscale $\left(\mathrm{H}_{2} \mathrm{O}\right)_{n}^{-}$clusters with $n=200,500,1000$ and 8000 with internal nuclear kinetic energies consistent with an equilibrium distribution at $T=200 \mathrm{~K}$. The simulation methods are described in more detail elsewhere. ${ }^{33}$ The water molecules are described classically, interacting via a flexible three-site potential, whereas the electron is represented by its wavefunction in a plane-wave basis evenly distributed on a $32 \times 32 \times 32$ point grid for $n=200,500$, and 1000 , and on a $16 \times 16 \times 16$ point grid for $n=8000$. The $32 \times 32 \times 32$ grid spanned $36.34 \AA$. The smaller grid for the $n=8000$ case (16×16×16 grid spanning $18.17 \AA$ ) was used to save substantial CPU time. Due to the highly localized nature of the excess electron distribution, the use of the smaller grid did not affect the accuracy of the results. The water-electron interaction is modeled by an approximate pseudopotential model developed to describe the bulk hydrated electron. ${ }^{34}$ Hence, we expect the model to perform best for relatively large clusters. The water molecules evolve under the combined influence of other classical molecules and the adiabatically propagated ground state electron. In the simulation, the surface states and the internal states of the electron are identified by geometrical parameters: the radius of gyration of the excess electron $\left(r_{e}=\left\langle r^{2}\right\rangle^{1 / 2}\right)$, the radius of the cluster $\left(r_{\mathrm{c}}\right)$, and the distance between the centers of the two distributions $(R)$. We define an IB state if it is distinctly clear that $R+r_{\mathrm{e}}<r_{\mathrm{c}}$, while for a surface-bound state $R \sim r_{c} .^{13}$

The simulations are started from one of two distinctly different initial conditions: from clusters with preformed interior-bound excess electron states or from those with preformed surface electron states. The clusters with initial interior excess electron state are initiated from equilibrium bulk hydrated electron simulation structures (similar to previous work). ${ }^{13}$ The initial clusters with preformed surface 
states are prepared by using a confining potential (umbrella potential) which constrains the electron center of mass to the surface of the clusters. After preparing the initial configurations at the desired simulation temperature, removing the constraints, and equilibrating the clusters anions, molecular dynamics simulations are carried out in the microcanonical (constant NVE) ensemble. The simulations here have a typical duration of $50 \mathrm{ps,} \mathrm{and} \mathrm{we} \mathrm{use} \mathrm{the} \mathrm{term} \mathrm{"persistent"} \mathrm{to} \mathrm{describe} \mathrm{a} \mathrm{state}$ whose SB or IB initial character does not change during that time frame.

\section{Results}

Figure 1 shows the time evolution of the energy eigenvalues (ground state and first three excited states) of the electron in four different size clusters ( $n=200,500$, 1000 and 8000 ) at $200 \mathrm{~K}$. We observe that the excess electron in all four simulated cases persists in both interior and surface states at $200 \mathrm{~K}$. This situation is clearly different from the results of a previous simulation where we found that for the smaller clusters $(n=20,33,45,66$ and 104) the preformed initial interior state clusters isomerize spontaneously to surface state clusters at $200 \mathrm{~K} .^{13}$ It was found that only for much lower temperature simulations $(T=100 \mathrm{~K})$ do we observe persistent IB clusters for $n=45,66$ and 104, while the $n=20$ and 33 IB cluster anions relaxed to SB states at $100 \mathrm{~K}$ also. The difference in typical SB vs. IB energy gaps between ground and excited states is evident in Fig. 1, a point that will be explored more completely below.

Figure 2 plots the ground state excess electron energies of the four clusters examined here and those of previous work for smaller cluster sizes ${ }^{13}$ all at $200 \mathrm{~K}$. Because our model does not include explicitly polarizable water molecules, the negative of the electronic ground state energy is equivalent to the electron vertical 
detachment energy. The average ground state energies for the IB clusters smoothly become more stable from $-3.0 \mathrm{eV}(n=200)$ to $-3.9 \mathrm{eV}(n=8000)$. It is generally expected that the IB cluster physical properties extrapolate to those of a bulk hydrated state at infinite size. The ground state energy for the IB excess electron conforms to this tendency with a linear scaling to the inverse cube of the cluster size, $n^{-1 / 3}$, which is also predicted by continuum dielectric theory. ${ }^{11}$ The IB cluster energies simulated in this work extrapolate to an intercept of $-4.4 \mathrm{eV}$. This extrapolated value clearly exceeds the simulated ground state energy of an equilibrium hydrated electron at 300 $\mathrm{K}(-3.1 \mathrm{eV})$ reported previously by us using the same model. ${ }^{34}$ Although the physical properties of the hydrated electron exhibit some temperature dependence, one can expect only a slightly stronger excess electron stabilization at $200 \mathrm{~K} \cdot{ }^{35}$ The disagreement between bulk and extrapolated cluster ground state energies $(\sim 1 \mathrm{eV})$ can be attributed mainly to the neglect of the long range interactions in the bulk simulations, where the long range effects were smoothly truncated, ${ }^{34}$ while all interactions are explicitly included in the (finite) cluster calculations. To test this hypothesis, we performed a bulk hydrated electron simulation at $300 \mathrm{~K}$ using the Ewald method, ${ }^{36,37}$ and obtained $-3.9 \mathrm{eV}$ for the ground state energy of the hydrated electron in sensible agreement with the extrapolated value. We note that both the bulk hydrated electron ground state energy and the extrapolated IB cluster value simulated with this model are more negative than a value of $-3.3 \mathrm{eV}$ from extrapolation of the experimental VDE's of clusters. ${ }^{22}$

The trends are somewhat different for the clusters that support SB states. The ground state energy of the SB electrons is significantly less negative than that for their IB counterparts, slowly converging from $-1.9 \mathrm{eV}(n=200)$ to $-2.8 \mathrm{eV}(n=1000)$ to $3.6 \mathrm{eV}(n=8000)$. We note that the simulated SB data deviates distinctly from a 
linear dependence on $n^{-1 / 3}$ when all of the data is considered together. The origin of this behavior is considered in some detail below along with a consideration of continuum dielectric models for the system. The extrapolated, infinite size, limiting case of the simulated SB anions is an excess electron solvated at an infinite water/air interface. The present value for the largest cluster considered is once again significantly more negative (by $\sim 1 \mathrm{eV}$ ), than the result we reported for electron solvation at a supercooled $(T=200 \mathrm{~K})$ water/air interface $(-2.7 \mathrm{eV}) .{ }^{38}$ Here again, since the interfacial simulations used a finite cut-off, the discrepancy stems from the neglect of long-range interactions there. Since these differences in the ground state energies of the simulated limiting cases appear as a constant shift, this does not influence the main conclusions of either the present or previous works.

Figure 3 illustrates the major structural differences of the SB and IB forms in terms of characteristic geometrical parameters. The most obvious difference appears in the position of the center of the electron distribution relative to that of the molecular cluster. For surface states, the distance $(R)$ between the centers of the electron distribution and the center of mass of the cluster is comparable to the radius of the cluster $\left(r_{\mathrm{c}}\right), R \sim r_{c}$, while for interior states $R+r_{\mathrm{e}}<r_{\mathrm{c}}$. These relations are clearly reflected in Figure 3. In addition, the radius of gyration and the kinetic energy of the excess electron exhibit different trends with size in the two "isomers", as is more evident in Figure 4. Figure 4 collects the mean values of electron radius of gyration $r_{e}$ and kinetic energy $E_{k i n}$ for all clusters studied. $r_{e}$ is nearly invariant (2.3$2.4 \AA$ ) in the simulated IB clusters and is in good agreement with the computed bulk hydrated electron radius $(2.4 \AA) .{ }^{34}$ The SB states are noticeably more diffuse, and contract upon increasing the cluster size from $r_{e}=5.5 \AA$ down to $3.2 \AA$ for $n=20 \rightarrow$ 104, from previous work ${ }^{13}$, and $3.2 \AA$ down to $2.6 \AA$ for $n=200 \rightarrow 8000$. The radii 
for the SB electrons, thus, smoothly approach the radius of the interfacially solvated electron $(2.6 \AA)^{41}$ from above. This behavior, which has been recognized from the simulations of smaller clusters, ${ }^{13}$ completely parallels the trend for the radii extracted from the experimental cluster anion absorption spectra. ${ }^{30}$ As expected, a similar pattern holds for the kinetic energy of the excess electron, also shown in Fig. 4. The IB states have higher kinetic energy than the SB states, and all IB state kinetic energies are similar to one another, and distinctly larger than the large SB cluster limit. The kinetic energy increases with increasing cluster size for SB states approaching the bulk interface limit from below. The trend of the data follows the experimentally derived size dependence. ${ }^{30}$ Our present data, thus, provides a complete description with respect to size of these two characteristics and two distinct structural classes.

Next, we return to the ground state energy data in Fig. 2 and consider the source of the observed curvature in the size dependence of the electron energy/vertical detachment energy. This is effectively done by considering the results of a dielectric continuum model. One might, in fact, expect distinct deviation from linearity for SB states for a number of reasons. First, it has been shown ${ }^{39}$ that the present pseudopotential underestimates the electron-cluster interaction energies for the smallest clusters due to the neglect of electron-molecule dispersion interactions. ${ }^{12,40}$ Second, specific interactions (e.g., hydrogen bonds) of the nearest water molecules with the electron will weigh more heavily in the energy for the smaller clusters. In addition, even a continuum dielectric theory ${ }^{41}$ includes a dependence on the electron kinetic energy and radius, which we find varies with $n$ for SB states. We show, in Fig. 2, results obtained by alternative routes for both SB and IB states. We use the continuum model of Makov and Nitzan ${ }^{41}$, (SB states: eq. (23) and (28); IB states: eq. 
(22b) and (29) in Ref. 41) and extract the needed parameters from our simulations, or alternatively from experiment, ${ }^{30}$ for each available case. The parameters are electron radius, electronic kinetic energy, cluster radius, denoted here as $r_{e}, E_{k i n}, r_{c}$, and corresponding to $a, \Delta K, R$, respectively in Ref. 41 . The continuum results are shown as colored symbols in Fig. 2.

The dielectric theory for both SB and IB states is evidently rather linear on this plot. We find that the kinetic energy is quite linear in $n^{-1 / 3}$ (not shown), and that the variation in radius for SB states affects terms in a nearly compensating manner. For the simulated IB states, the result is that the continuum theory agrees closely with the simulation. For the SB states, the continuum theory is seen to converge toward the simulated results with increasing size, with substantial deviations at smaller cluster size $(n<100)$. However, we note that for $n<\sim 30$, the continuum results yield unbound energies, using the simulated kinetic energies, and yield energies considerably more positive than simulation when using experimentally inferred parameters. Hence, for the smaller clusters, lying in the experimentally currently determined range $(n<\sim 200)$, we infer that these deviations from continuum theory result from the limitations of that theory in describing the attractive energetics of water-electron interactions at short distances. As noted above and elsewhere, ${ }^{13}$ the simulations for the smallest cluster $(n=20)$ is likely the least accurate, but that does not affect these conclusions.

Finally, we have included in Fig. 2 the linear fit (dashed line) inferred from our previous data for SB states $(n \leq 104) .{ }^{13}$ We note that this line also adequately describes the new data for $n=200$, and only deviates glaringly from linearity for substantially larger $n$. The extrapolated SB ground state energy at infinite cluster size is in reasonably good accord with the experimental extrapolation of $3.3 \mathrm{eV}{ }^{22}$ The 
reasonable agreement of our simulations with the continuum theory for large $n$, and the reasonable agreement of the simulations with experiment for modest $n$, together suggest again ${ }^{13}$ that the experimental data for these smaller clusters $(n<200)$ reports the behavior of SB states.

The absorption spectra of the anions are shown in Figure 5. We observe that the spectra of the clusters with IB excess electron state appear slightly blue shifted from the $300 \mathrm{~K}$ bulk hydrated electron spectrum (maximum at $1.72 \mathrm{eV}$ experimentally, ${ }^{42} 1.92 \mathrm{eV}$ from simulation ${ }^{34}$ ), mainly due to electrostriction. ${ }^{13}$ The surface-bound state spectra are, on the other hand, significantly red-shifted, gradually blue shifting with increasing size to larger energy gaps in apparent agreement with experiments. ${ }^{5}$ The maximum of the absorption spectra smoothly approaches the simulated spectrum of an excess electron at an infinite water/air interface, which appears with a maximum at $1.5 \mathrm{eV}$ for a $T=200 \mathrm{~K}$ liquid water/air interface. The positions of the maxima of the calculated SB cluster spectra extrapolate to a comparable value based only on the smaller cluster data, $1.7 \pm 0.3 \mathrm{eV} \cdot{ }^{13,38}$ We also note that the surface state spectrum clearly does not extrapolate to the bulk hydrated electron spectrum in our data.

\section{Discussion and Conclusions}

In the calculations presented, we have evaluated the results expected for the vertical detachment energy, optical absorption spectrum, and electronic properties for both surface-bound and interior-bound excess electron states as a function of cluster size at a nominal temperature of $200 \mathrm{~K}$. The results now span the entire range of $n$ for both SB and IB cases. 
These results for SB and IB states show that the electronic ground state energies (which estimate detachment energies) are both relatively linear functions of inverse cluster radius $\left(r_{\mathrm{c}} \sim n^{-1 / 3}\right)$ with similar slopes, but different infinite cluster intercepts. When limited to only modest cluster sizes $(n \leq 200)$, the SB series extrapolates to a value reasonably close to that obtained by extrapolation of experimental cluster results. ${ }^{22}$ However, deviations from linearity for the simulated SB state energies become very evident when large clusters are considered and the limiting value at an infinite interface is considerably more negative, $\sim-4 \mathrm{eV}$ in our model. In parallel, the SB state electronic radii monotonically approach their limit from above with increasing cluster size, while the kinetic energy of the excess electron increases with increasing cluster size, approaching the limit from below. Both of these trends are in accord with experiment. ${ }^{30}$ In contrast, the IB states have an electron size and kinetic energy which are essentially independent of cluster size over an enormous range. Correspondingly, the absorption spectra of SB states evolve continuously to the blue with cluster size, while those for IB states are essentially insensitive to cluster size.

Consideration of the dielectric continuum model of Makov and Nitzan ${ }^{41}$ using values of physical parameters taken from the simulations shows that the IB states follow that model closely ( $n \geq 200)$, while the SB states and continuum model converge well only at large cluster size $(n>500)$. The fact that the simulated and continuum models deviate from one another in magnitude and slope at smaller size ( $\mathrm{n}$ $<\sim 200)$ for SB states is assigned to an underestimate by the continuum model of the attractive interaction of electron and water at short range, a result that is sensible.

A particularly interesting simulation result from the present study is the mere persistence of both interior and surface-bound excess electron states at $200 \mathrm{~K}$. It is 
especially instructive to take a look at the temperature and size dependence of these persisting states. To extend our examinations to higher temperature and complete our previous investigations on smaller clusters ${ }^{13}$, we have also attempted to simulate large surface binding cluster anions at $300 \mathrm{~K}$. However, with the exception of the $n=200$ anion, larger preformed SB cluster anions at $300 \mathrm{~K}$ relaxed spontaneously in a short time to interior binding cluster anion. We summarize our collected observations on persisting states in Table 1. Clearly, the largest clusters at around room temperature are stable in an IB state $(n>200, T=300 \mathrm{~K})$, while the smallest clusters $(n=20,33)$ are stable in SB electron states regardless of the temperature. The surface-bound states remain dominant at higher temperatures up to around $n=200$ cluster size. Above this size, a prediction of which form becomes thermodynamically favored will require explicit free-energy calculations for those clusters where both states can persist. Thus, the question of where, in size and temperature, the SB-IB transition occurs is not yet fully clarified. Nevertheless, the structure of the table entries suggests the presence of a modest free energy barrier between SB and IB states which grows with cluster size for small clusters. The role of such a barrier in the formation kinetics of anionic clusters experimentally is an aspect worthy of further examination.

The experimentally observed sequences of related cluster "isomers" clearly manifest more than two classes of cluster. ${ }^{9}$ Hence, the issue is more complex than SB vs. IB, and further considerations are necessary for a satisfactory solution. We have considered only equilibrium systems with a characteristic temperature as the starting point of the simulations. The examination of non-equilibrium distributions seems a necessary element to achieve agreement with this broad set of observations. In particular, the preparation method of the clusters may have an important effect on the 
physical properties. ${ }^{31}$ In a separate work, we shall investigate the observables following from alternative preparation histories. 


\section{Acknowledgements}

L.T. gratefully acknowledges the tenure of the Öveges Research Fellowship. The work was supported by a grant to L. T. from the National Research Fund of Hungary (OTKA) under Contracts No. T049715 and IN69707. P. J. R. is grateful to the National Science Foundation (CHE-0615173) and the R. A. Welch Foundation (F0019) for support of this work. 


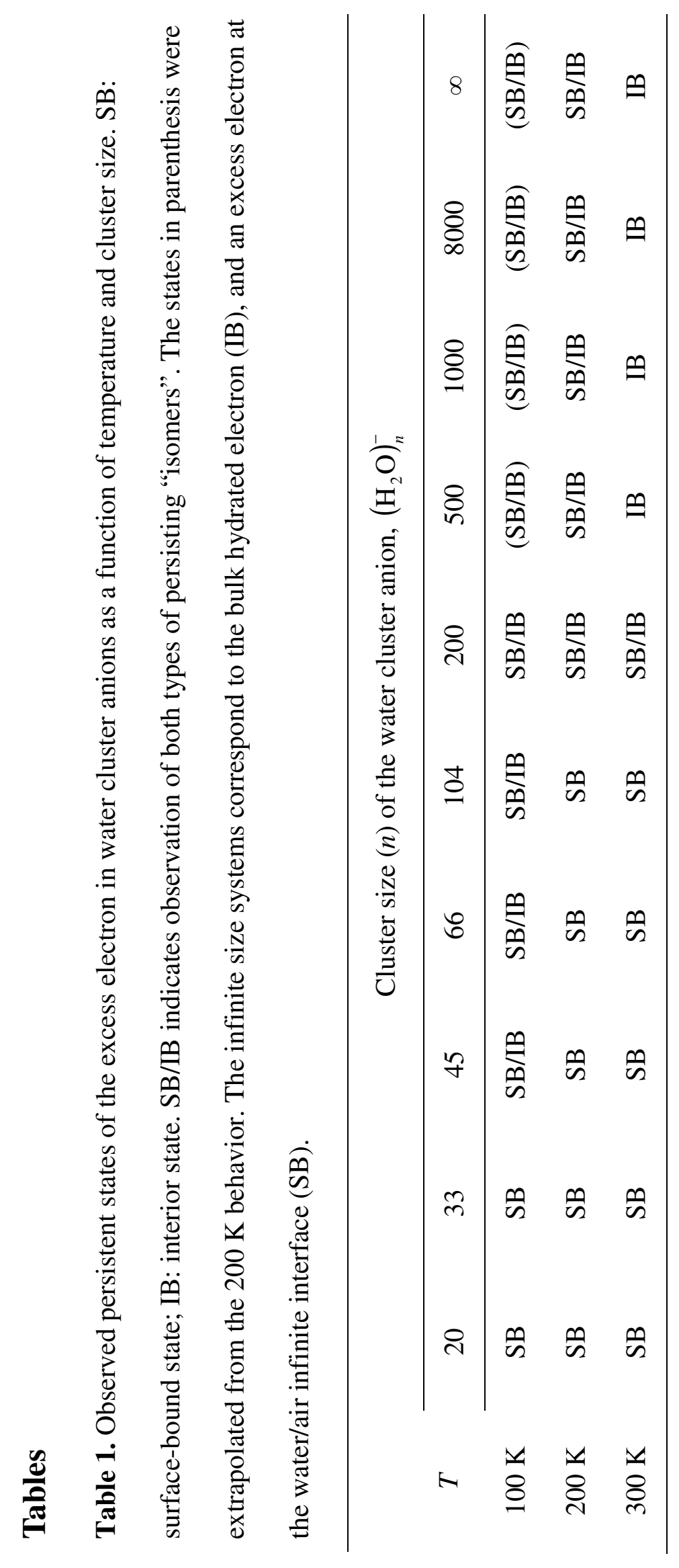




\section{Figure Captions}

Figure 1. Time evolution of the equilibrium ground state energy and the first three excited state energies of the excess electron in water cluster anions at a nominal temperature of 200 $\mathrm{K}$. Left column is for SB excess electron states, right column is for IB states.

Figure 2. Ground state energies at $200 \mathrm{~K}$ of surface bound (SB) cluster anions (squares) and interior bound (IB) cluster anions (triangles). Black symbols show the simulated results. Red symbols represent the energies calculated from a dielectric continuum theory (Ref. 41) using our simulated values for the electron radius, electron kinetic energy, water cluster radius. The dashed line indicates the line fit to the simulated data in previous work (five smallest clusters, $n=20-104) .{ }^{13}$ Green symbols show the dielectric continuum theory computed instead using the values of $r_{e}$ and $E_{k i n}$ inferred from experimental data. ${ }^{30}$ For all points, the high frequency dielectric constant is set to unity, in accord with our simulated model.

Figure 3. Time evolution of the equilibrium geometric parameters of water cluster anions at a nominal $200 \mathrm{~K}$. Left column is for SB excess electron states, right column is for IB states. Dotted line: radius of gyration of the excess electron; dashed line: radius of gyration of the cluster; solid line: distance between the centers of the electron and the cluster.

Figure 4. Radius of gyration $r_{e}$ and kinetic energy $E_{\text {kin }}$ of the excess electron at $200 \mathrm{~K}$ for surface binding SB cluster anions (squares) and interior binding IB cluster anions (triangles). Filled symbols show the present work, open symbols from previous work $(n=20,33,45$, 66 104). ${ }^{13}$ The insets show part of the data on an expanded scale.

Figure 5. Equilibrium optical absorption spectra of water cluster anions at a nominal $200 \mathrm{~K}$. Top panel: IB states ( $n=200$ (dash-dot), 500 (dash), 1000 (dash), 8000 (solid); bottom panel: SB states ( $n=45$ (dash-dot), 200 (dash), 1000 (dash), 8000 (solid). $n=45$ data from previous work. $^{13}$ 
Figure 1. Madarász, Rossky and Turi

\section{SB states IB states}

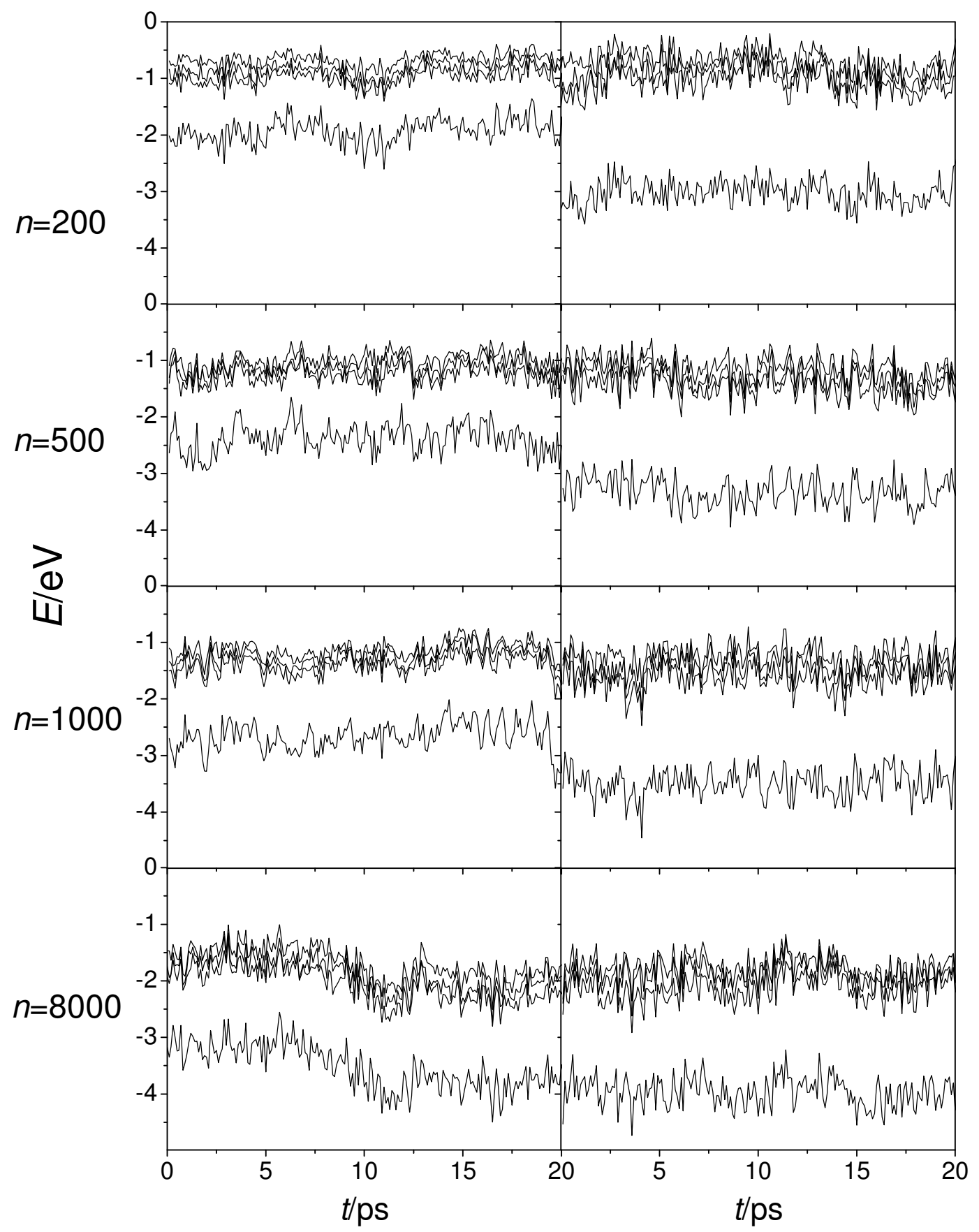


Figure 2. Madarász, Rossky and Turi

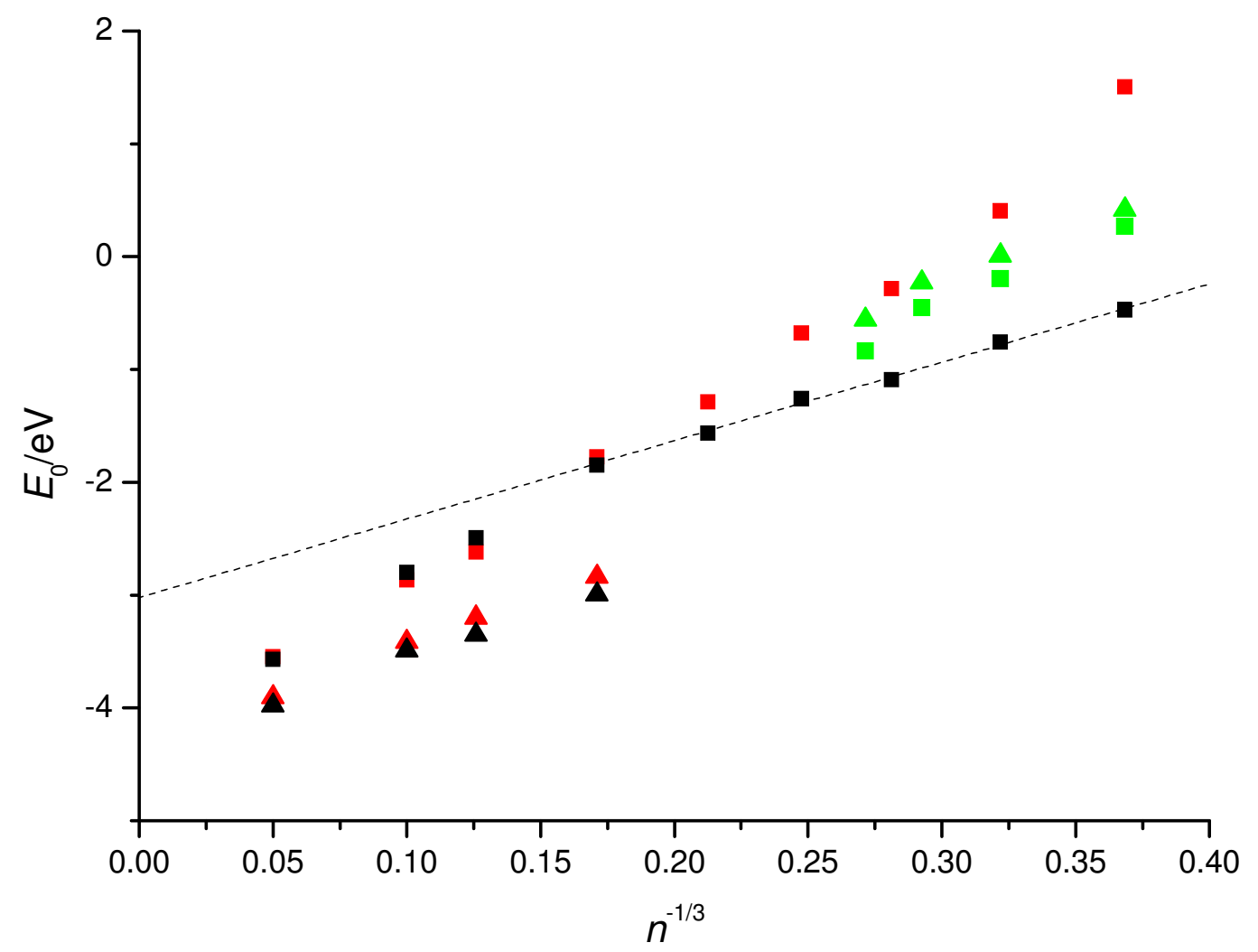


Figure 3. Madarász, Rossky and Turi

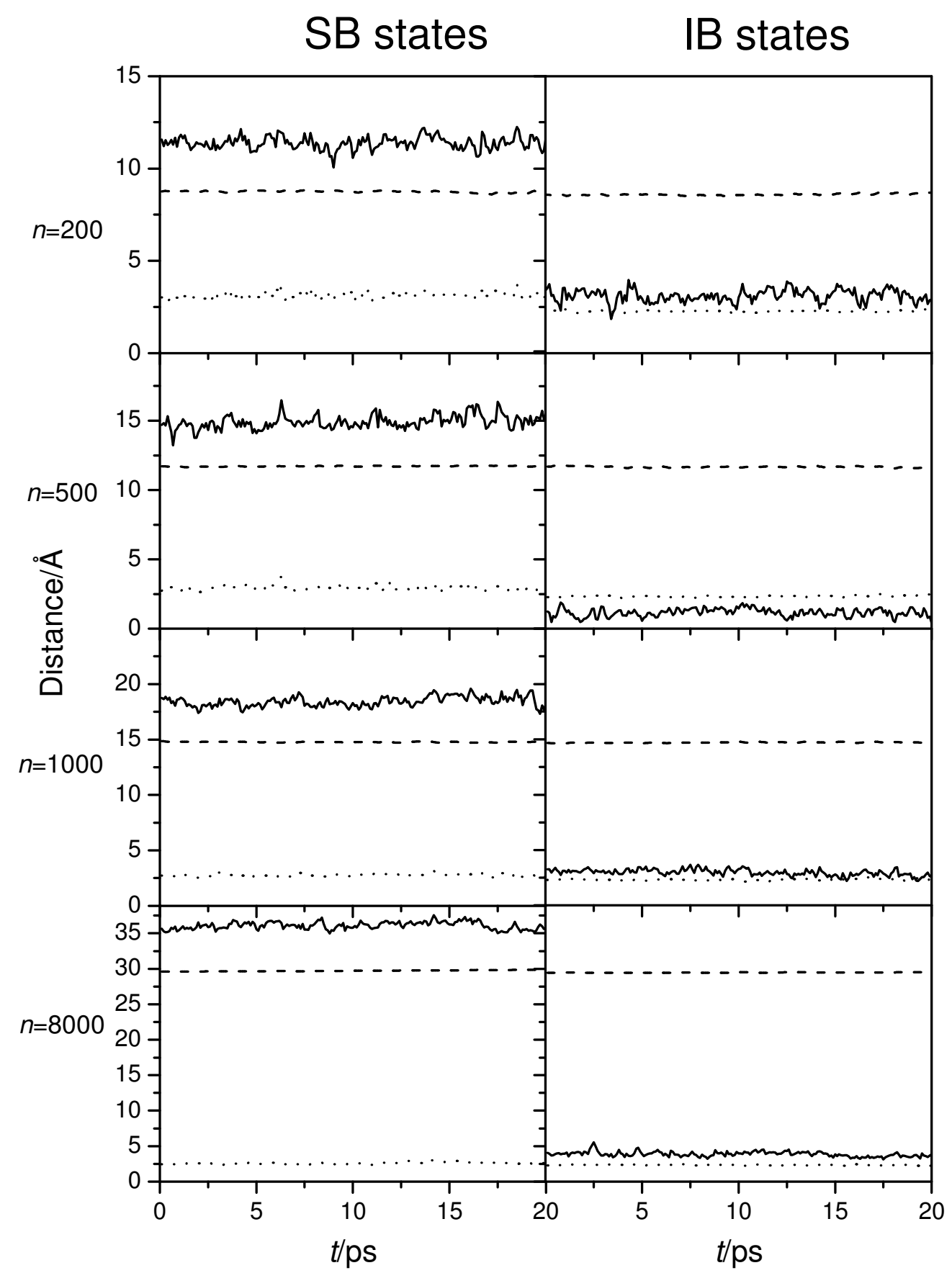


Figure 4. Madarász, Rossky and Turi

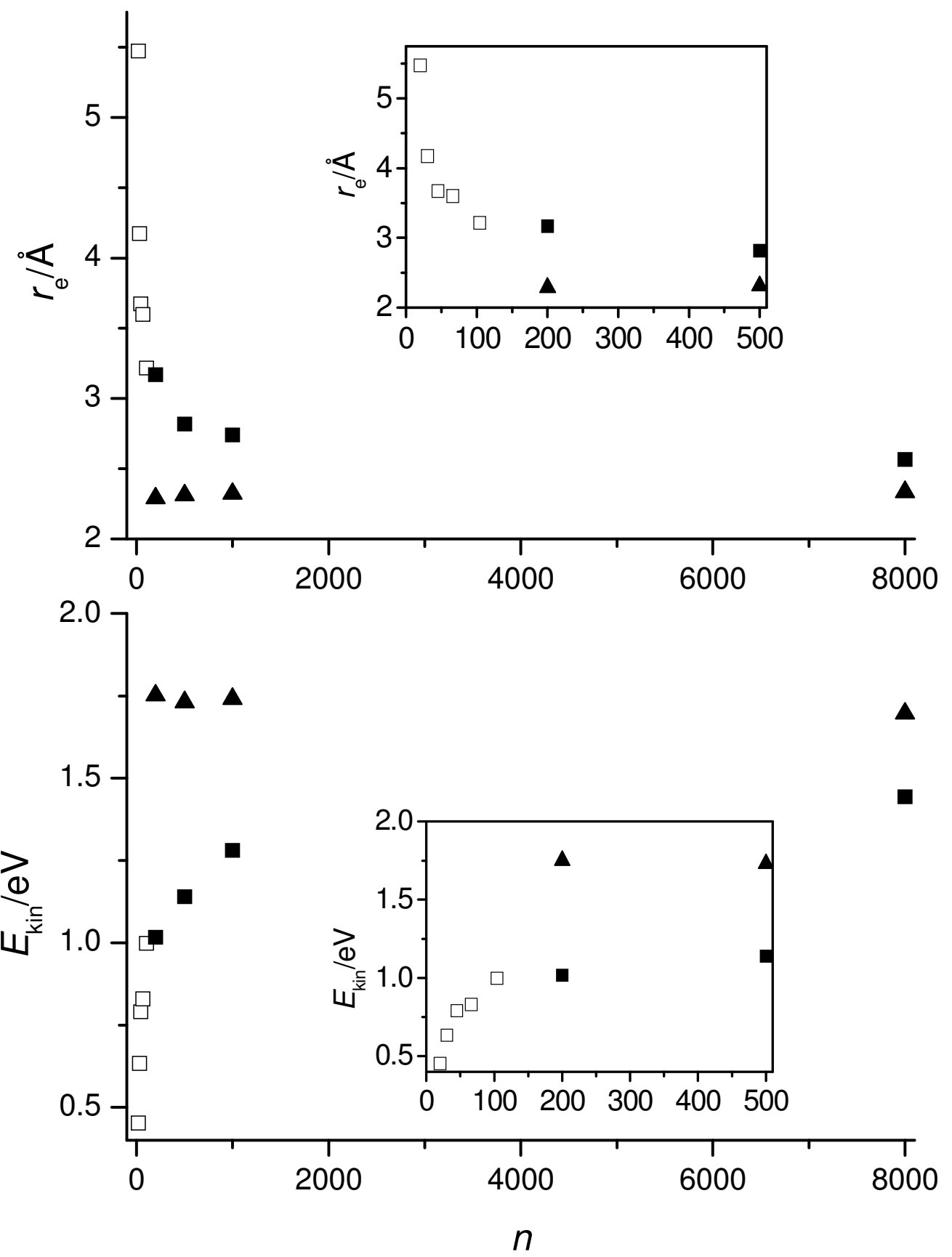


Figure 5. Madarász, Rossky and Turi
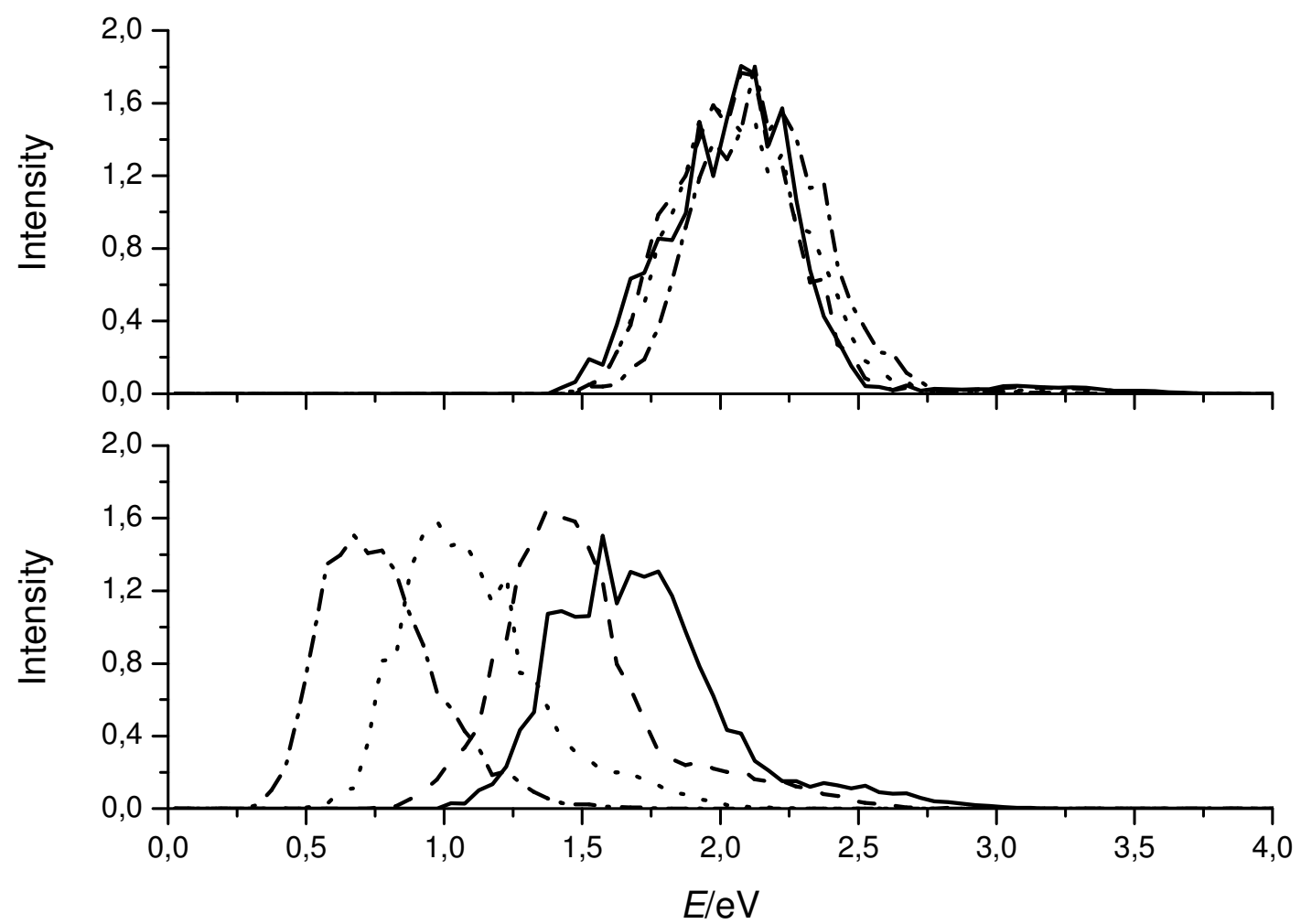


\section{References}

${ }^{1}$ C. Garrett, D. A. Dixon, D. M. Camaioni, D. M. Chipman, M. A. Johnson, C. D. Jonah, G. A. Kimmel, J. H. Miller, T. N. Rescigno, P. J. Rossky, S. S. Xantheas, S. D. Colson, A. H. Laufer, D. Ray, P. F. Barbara, D. M. Bartels, K. H. Becker, K. H. Bowen, Jr., S. E. Bradforth, I. Carmichael, J. V. Coe, L. R. Corrales, J. P. Cowin, M. Dupuis, K. B. Eisenthal, J. A. Franz, M. S. Gutowski, K. D. Jordan, B. D. Kay, J. A. LaVerne, S. V. Lymar, T. E. Madey, C. W. McCurdy, D. Meisel, S. Mukamel, A. R. Nilsson, T. M. Orlando, N. G. Petrik, S. M. Pimblott, J. R. Rustad, G. K. Schenter, S. J. Singer, A. Tokmakoff, L.-S. Wang, C. Wittig, and T. S. Zwier, Chem. Rev. 105, 355 (2005).

${ }^{2}$ H. Haberland, H. G. Schindler and D. R. Worsnop, Ber. Bunsenges. Phys. Chem. 88, 270 (1984).

${ }^{3}$ M. Knapp, O. Echt, D. Kreisle and E. Recknagel, J. Phys. Chem. 91, 2601 (1987).

${ }^{4}$ J. V. Coe, G. H. Lee, J. G. Eaton, S. T. Arnold, H. W. Sarkas and K. H. Bowen, J. Chem. Phys. 92, 3980 (1990).

${ }^{5}$ P. Ayotte, and M. A. Johnson, J. Chem. Phys. 106, 811 (1997).

${ }^{6}$ A. E. Bragg, J. R. R. Verlet, A. Kammrath, O. Cheshnovsky, and D. M. Neumark, Science 306, 669 (2004).

${ }^{7}$ D. H. Paik, I-R. Lee, D.-S. Yang, J. S. Baskin, and A. H. Zewail, Science 306, 672 (2004).

${ }^{8}$ N. I. Hammer, J.-W. Shin, J. M. Headrick, E. G. Diken, J. R. Roscioli, G. H. Weddle, and M. A. Johnson, Science 306, 675 (2004).

${ }^{9}$ J. R. R. Verlet, A. E. Bragg, A. Kammrath, O. Cheshnovsky, and D. M. Neumark, Science 307, 93 (2005). 
${ }^{10}$ F. Zappa, S. Denifl, I. Mähr, A. Bacher, O. Echt, T. D. Märk, and P. Scheier, J. Am. Chem. Soc, 130, 5573 (2008).

${ }^{11}$ R. N. Barnett, U. Landman, C. L. Cleveland, and J. Jortner, J. Chem. Phys. 88, 4429 (1988).

${ }^{12}$ K. D. Jordan, Science 306, 618 (2004).

${ }^{13}$ L. Turi, W.-S. Sheu, and P. J. Rossky, Science 309, 914 (2005).

${ }^{14}$ J. M. Herbert, and M. Head-Gordon, Phys. Chem. Chem. Phys. 8, 68 (2006).

${ }^{15}$ J. M. Herbert, and M. Head-Gordon, J. Phys. Chem. A 109, 5217 (2005).

${ }^{16}$ T. Sommerfeld, and K. D. Jordan, J. Am. Chem. Soc. 128, 5828 (2006).

${ }^{17}$ A. Khan, J. Chem. Phys. 125, 025307 (2006).

${ }^{18}$ A. Khan, J. Chem. Phys. 126, 027102 (2006).

${ }^{19}$ T. Sommerfeld, J. Chem. Phys. 126, 027101 (2006).

${ }^{20}$ T. Frigato, J. VandeVondele, B. Schmidt, C. Schütte, and P. Jungwirth, J. Phys. Chem. A, 112, 6125 (2008).

${ }^{21}$ J. R. R. Verlet, A. E. Bragg, A. Kammrath, O. Cheshnovsky, and D. M. Neumark, Science 310, 1769 (2005).

${ }^{22}$ J. V. Coe, A. D. Earhart, M. H. Cohen, G. J. Hoffman, H. W. Sarkas, and K. H. Bowen, J. Chem. Phys. 107, 6023 (1997).

${ }^{23}$ J. V.Coe, Int. Rev. Phys. Chem. 20, 33 (2001).

${ }^{24}$ N. I. Hammer, J. R. Roscioli, and M. A. Johnson, J. Phys. Chem. A 109, 7896 (2005).

${ }^{25}$ J. V. Coe, S. T. Arnold, J. G. Eaton, G. H. Lee and K. H. Bowen, J. Chem. Phys. 125, $014315(2006)$. 
${ }^{26}$ A. Kammrath, J. R. R. Verlet, G. B. Griffin, and D. M. Neumark, J. Chem. Phys. 125, $076101(2006)$

${ }^{27}$ K. R. Asmis, G. Santambrogio, J. Zhou, E. Garand, J. Headrick, D. Goebbert, M. A. Johnson, and D. M. Neumark, J. Chem. Phys. 126, 191105 (2007).

${ }^{28}$ J. V. Coe, S. M. Williams, and K. H. Bowen, Int. Rev. Phys. Chem. 27, 27 (2008).

${ }^{29}$ L. Turi, W.-S. Sheu, and P. J. Rossky, Science 310, 1769 (2005).

${ }^{30}$ D. M. Bartels, J. Chem. Phys. 115, 4404 (2001).

${ }^{31}$ M. Mitsui, S. Kokubo, N. Ando, Y. Matsumoto, A. Nakajima and K. Kaya, J. Chem. Phys. 121, 7553 (2004).

${ }^{32}$ P. J. Campagnola, L. A. Posey and M. A. Johnson, J. Chem. Phys. 95, 7998 (1991).

${ }^{33}$ F. A. Webster, P. J. Rossky and R. A. Friesner, Comput. Phys. Commun. 63, 494 (1991).

${ }^{34}$ L. Turi, and D. Borgis, J. Chem. Phys. 117, 6186 (2002).

${ }^{35}$ C. Nicolas, A. Boutin, B. Lévy and D. Borgis, J. Chem. Phys. 118, 9689 (2003).

${ }^{36}$ M. P. Allen and D. J. Tildesley, Computer Simulation of Liquids (Clarendon, Oxford, 1987).

${ }^{37}$ C.-Y. Yang, K. F. Wong, M. S. Skaf, and P. J. Rossky, J. Chem. Phys. 114, 3598 (2001).

38 Á. Madarász, P. J. Rossky, and L. Turi, J. Chem. Phys. 126, 234707 (2007).

${ }^{39}$ L. Turi, Á. Madarász, and P. J. Rossky, J. Chem. Phys. 125, 014308 (2006).

${ }^{40}$ F. Wang, and K. D. Jordan, Ann. Rev. Phys. Chem. 54, 367 (2003).

${ }^{41}$ G. Makov, and A. Nitzan, J. Phys. Chem. 98, 3459 (1994).

${ }^{42}$ F.-Y. Jou, G. R. Freeman, J. Phys. Chem. 81, 909 (1977). 